\title{
Effect of Different Packing and Storage Conditions on Biochemical Parameters in Extending the Vase Life of Cut Carnation cv. Kiro
}

\author{
P. Pranuthi, T. Suseela, D.V. Swami*, D.R. Salomi Suneetha and V. Sudha Vani
}

Department of Floriculture and Landscape Architecture, Dr. Y.S.R. Horticultural University, Venkataramannagudem, West Godavari dist. (Andhra Pradesh), India

*Corresponding author

\section{A B S T R A C T}

\section{Keywords}

Carnation, Packing,

Storage conditions,

Chlorophyll content and

ethylene estimation

Article Info

Accepted:

10 July 2018

Available Online:

10 August 2018
An experiment was carried out to study the effect of different packing and storage conditions on vase life of cut carnation cv. Kiro. The flowers packed in polypropylene at $5^{\circ} \mathrm{C}$ cold storage under wet condition recorded significantly highest total chlorophyll content of leaf (78.98 SPAD unit), highest total chlorophyll content of calyx (38.00 SPAD unit) and lowest ethylene estimation $(0.27 \mathrm{ppm} / \mathrm{h})$ which have extended the vase life period of carnation flower cv. Kiro.

\section{Introduction}

Carnation (Dianthus caryophyllus L.) belongs to the family caryophyllaceae and native to Mediterranean region. Carnation is one among the top five cut flowers in the world and in India (Singh, 2006). The beauty of the cut flowers lies with the freshness of the flowers for longer time without post-harvest losses.

It is claimed that 70 per cent of the potential quality of cut flowers is pre-determined at harvest, whereas the remaining 30 per cent is influenced by post-harvest factors. All along the marketing channel, there is enormous loss in value of cut flowers which could be of 50 per cent of farm value (Bhattacharjee, 1999).
Post-harvest life of cut flowers also depends upon efficient packaging and storage. Appropriate packaging of cut flowers together with pulsing are helpful to ensure fresh quality of flowers. Packing is a tool for controlling flower quality in the distribution chain. Apart from preventing mechanical damage, the package serves as a barrier between the conditions inside and outside the package. It protects the flowers from unfavorable outside conditions and enables a micro-climate to develop inside the package (Lavanya et al., 2016). Packaging must ensure protection of flowers against flower damage, water loss and external conditions, which are detrimental to flowers in transit (Sivaswamy et al., 1999). Storage at low temperature under wet 
conditions results in low metabolic activities like respiration, transpiration and maintains high humidity and increased cell turgidity and cell enlargement there by keeping the flower quality and increased vase life (Halevy and Mayak, 1981).

\section{Materials and Methods}

The experiment was held at laboratory of Floriculture and Landscape Architecture, College of Horticulture, Dr. Y.S.R. Horticultural University, Venkataramannagudem, West Godavari dist (A.P) during year 2017-18. Experiment laid out in completely randomised design under factorial concept. The total number of treatment combinations is 20. They are as follows $\mathrm{T}_{1}-\mathrm{P}_{1} \mathrm{~S}_{1}$ : PVC cling film + Ambient temperature $\left(22 \pm 2^{\circ} \mathrm{C}\right)$ (Wet storage), $\mathrm{T}_{2}-\mathrm{P}_{2} \mathrm{~S}_{1}$ : Polyethylene $(100$ gauge $)+$ Ambient temperature $\left(22 \pm 2^{\circ} \mathrm{C}\right)$ (Wet storage), $\mathrm{T}_{3}-\mathrm{P}_{3} \mathrm{~S}_{1}$ : Cellophane paper + Ambient temperature $\left(22 \pm 2^{\circ} \mathrm{C}\right.$ ) (Wet storage), $\mathrm{T}_{4}-\mathrm{P}_{4} \mathrm{~S}_{1}$ : Polypropylene + Ambient temperature $\left(22 \pm 2^{\circ} \mathrm{C}\right)$ (Wet storage), $\mathrm{T}_{5}-\mathrm{P}_{5} \mathrm{~S}_{1}$ : Control (open) + Ambient temperature $\left(22 \pm 2^{\circ} \mathrm{C}\right.$ ) (Wet storage), $\mathrm{T}_{6}-\mathrm{P}_{1} \mathrm{~S}_{2}$ : $\mathrm{PVC}$ cling film + Cold storage of $5^{\circ} \mathrm{C}$ (Wet storage), $\mathrm{T}_{7}-\mathrm{P}_{2} \mathrm{~S}_{2}$ : Polyethylene (100 gauge) + Cold storage of $5^{\circ} \mathrm{C}$ (Wet storage), $\mathrm{T}_{8}-\mathrm{P}_{3} \mathrm{~S}_{2}$ : Cellophane paper + Cold storage of $5^{\circ} \mathrm{C}$ (Wet storage), $\mathrm{T}_{9}-\mathrm{P}_{4} \mathrm{~S}_{2}$ : Polypropylene + Cold storage of $5^{\circ} \mathrm{C}$ (Wet storage), $\mathrm{T}_{10}-\mathrm{P}_{5} \mathrm{~S}_{2}$ : Control (open) + Cold storage of $5^{\circ} \mathrm{C}$ (Wet storage), $\mathrm{T}_{11}-\mathrm{P}_{1} \mathrm{~S}_{3}$ : PVC cling film + Ambient temperature $\left(22 \pm 2^{\circ} \mathrm{C}\right)$ (Dry storage), $\mathrm{T}_{12}-\mathrm{P}_{2} \mathrm{~S}_{3}$ : Polyethylene (100 gauge) + Ambient temperature $\left(22 \pm 2^{\circ} \mathrm{C}\right)$ (Dry storage), $\quad \mathrm{T}_{13}-\mathrm{P}_{3} \mathrm{~S}_{3}$ : Cellophane paper + Ambient temperature $\left(22 \pm 2^{\circ} \mathrm{C}\right)$ (Dry storage), $\mathrm{T}_{14}-\mathrm{P}_{4} \mathrm{~S}_{3}$ : Polypropylene + Ambient temperature $\left(22 \pm 2^{\circ} \mathrm{C}\right.$ ) (Dry storage), $\mathrm{T}_{15}-\mathrm{P}_{5} \mathrm{~S}_{3}$ : Control (open) + Ambient temperature $\left(22 \pm 2^{\circ} \mathrm{C}\right)$ (Dry storage), $\mathrm{T}_{16}-\mathrm{P}_{1} \mathrm{~S}_{4}$ : PVC cling film + Cold storage of $5^{\circ} \mathrm{C}$ (Dry storage), $\mathrm{T}_{17^{-}}$ $\mathrm{P}_{2} \mathrm{~S}_{4}$ : Polyethylene (100 gauge) + Cold storage of $5^{\circ} \mathrm{C}$ (Dry storage), $\mathrm{T}_{18}-\mathrm{P}_{3} \mathrm{~S}_{4}$ : Cellophane paper + Cold storage of $5^{\circ} \mathrm{C}$ (Dry storage), $\mathrm{T}_{19}-\mathrm{P}_{4} \mathrm{~S}_{4}$ : Polypropylene + Cold storage of $5^{\circ} \mathrm{C}$ (Dry storage), $\mathrm{T}_{20}-\mathrm{P}_{5} \mathrm{~S}_{4}$ : Control (open) + Cold storage of $5^{\circ} \mathrm{C}$ (Dry storage). The flowers were kept under dry and wet conditions under both cold storage at $5^{\circ} \mathrm{C}$ as well as ambient temperature $\left(22 \pm 2^{\circ} \mathrm{C}\right)$ by wrapping with different packing materials till the end of vase life period. For physical, physiological, biochemical and microbial studies, same treatments were repeated separately for destructive sampling. Therefore under wet conditions distilled water was used in experimentation. In each conical flask, six flowers were placed and considered as one replication. The treatments were replicated thrice. The individual flower stalks were placed randomly in $500 \mathrm{ml}$ conical flasks containing $300 \mathrm{ml}$ of distilled water under wet conditions. Observations were recorded changes in chlorophyll content of leaf, chlorophyll content of calyx and ethylene estimation.

Chlorophyll content in leaf and calyx was measured using chlorophyll meter SPAD-502. Amount of chlorophyll was expressed as SPAD units. All the flower spikes per treatment were packed separately in air tight package then sealed and the level of ethylene evolved was measured after one hour by ethylene analyser.

\section{Results and Discussion}

The total chlorophyll content of leaf was significant among all the treatment combinations. The highest total chlorophyll content of leaf (78.98 SPAD units) was recorded with flowers packed in polypropylene at $5^{\circ} \mathrm{C}$ cold storage under wet condition $\left(\mathrm{T}_{9}\right)$ which was on par with $\left(\mathrm{T}_{7}\right)$ and $\left(\mathrm{T}_{8}\right)$ flowers packed in polyethylene (100 gauge) at $5^{\circ} \mathrm{C}$ cold storage) under wet condition (77.27 SPAD units) and flowers 
packed in cellophane paper at $5^{\circ} \mathrm{C}$ cold storage under wet condition (76.42 SPAD units). Control (without packing) at ambient temperature $\left(22 \pm 2^{\circ} \mathrm{C}\right)$ under dry condition $\left(\mathrm{T}_{15}\right)$ recorded significantly the lowest total chlorophyll content of leaf (34.31 SPAD units) whereas, the remaining all other treatments recorded intermediate values. There were significant differences in total chlorophyll content of leaf during different days of vase life period. The chlorophyll content of leaf significantly decreased from $2^{\text {nd }}$ day (73.55 SPAD units) to $4^{\text {th }}$ day $(64.02$ SPAD units) at each successive interval of observation. The interaction effect between treatments and days on chlorophyll content of leaf was found significant. The treatment $\left(\mathrm{T}_{9}\right)$ flowers packed in polypropylene at $5^{\circ} \mathrm{C}$ cold storage under wet condition recorded significantly the highest chlorophyll content of leaf (79.85 SPAD units) on $2^{\text {nd }}$ day. On $4^{\text {th }}$ day polypropylene packed flowers at $5^{\circ} \mathrm{C}$ cold storage under wet condition $\left(\mathrm{T}_{9}\right)$ recorded the highest chlorophyll content of leaf (78.11 SPAD units). Flower packed in polypropylene at $5^{\circ} \mathrm{C}$ cold storage under wet condition $\left(\mathrm{T}_{9}\right)$ recorded the highest total chlorophyll content of leaf on all the days of vase life study. The increased chlorophyll content of leaf might be due to better water relations in flower stalk during earlier stages of experimentation helps in stabilization of cell organelle including chloroplast, whereas improper water relations and higher levels of electrical conductivity led to loss of membrane integrity resulting in damage of cell organelles including chloroplast in leaf as well as in calyx led to decreased chlorophyll content. The chlorophyll content was intensified when the flower dry matter content was higher and then it was faded due to depletion and damage of the chloroplast in the calyx and leaf at advanced senescence. These results were in the line with findings of Misson et al., (2000) where decreased chlorophyll content in leaves of carnation at increased storage period was observed. Similar results were also observed by Genkov et al., (1997) in flower crops, Sudha (2010) and Madhuri et al., (2016) in carnation.

The total chlorophyll content of calyx was significant among all the treatment combinations. The highest chlorophyll content of calyx (38.00 SPAD units) was recorded with flowers packed in polypropylene at $5^{\circ} \mathrm{C}$ cold storage under wet condition $\left(\mathrm{T}_{9}\right)$ which was followed by flowers packed in polyethylene (100 gauge) at $5^{\circ} \mathrm{C}$ cold storage under wet condition $\left(\mathrm{T}_{7}\right)$ (35.97 SPAD units). Control (without packing) at ambient temperature $\left(22 \pm 2^{\circ} \mathrm{C}\right)$ under dry condition $\left(\mathrm{T}_{15}\right)$ recorded significantly the lowest chlorophyll content of calyx (11.76 SPAD units) whereas, the remaining all other treatments recorded intermediate values. There were significant differences in chlorophyll content of calyx during different days of vase life period. The chlorophyll content of calyx significantly decreased from $2^{\text {nd }}$ day (29.97 SPAD units) to $4^{\text {th }}$ day $(25.77$ SPAD units) at each successive interval of observation. The interaction effect between treatments and days on chlorophyll content of calyx was found significant. The treatment $\left(\mathrm{T}_{9}\right)$ flowers packed in polypropylene at $5^{\circ} \mathrm{C}$ cold storage under wet condition recorded significantly the highest chlorophyll content of calyx (38.89 SPAD units) on $2^{\text {nd }}$ day. On $4^{\text {th }}$ day polypropylene packed flowers at $5^{\circ} \mathrm{C}$ cold storage under wet condition $\left(\mathrm{T}_{9}\right)$ recorded the highest chlorophyll content of calyx (37.12 SPAD units). Flowers packed in polypropylene at $5^{\circ} \mathrm{C}$ cold storage under wet condition $\left(\mathrm{T}_{9}\right)$ recorded the highest chlorophyll content of calyx on all the days of vase life study. The chlorophyll content of calyx increased initially and then decreased gradually and this might be due to increased chlorophyllase activity during initial days because of better water relations in flower stalk (Table 1-3). 
Table.1 Effect of different packaging and storage conditions on total chlorophyll content of leaf (SPAD units) during vase life of cut carnation (Dianthus caryophyllus L.) cv. Kiro

\begin{tabular}{|c|c|c|c|c|c|c|c|c|c|c|c|c|c|}
\hline \multirow[t]{2}{*}{ Treatments } & \multicolumn{13}{|c|}{ Days } \\
\hline & 2 & 4 & Mean & 6 & 8 & 10 & 12 & 14 & 16 & 18 & 20 & 22 & 24 \\
\hline $\mathrm{T}_{1}-\mathrm{P}_{1} \mathrm{~S}_{1}$ & 71.69 & 66.59 & 69.14 & 63.89 & - & - & - & - & - & - & - & - & - \\
\hline$\overline{\mathbf{T}_{2}-\mathbf{P}_{2} \mathbf{S}_{1}}$ & 72.84 & 68.76 & 70.80 & 65.41 & - & - & - & - & - & - & - & - & - \\
\hline $\mathbf{T}_{3}-\mathbf{P}_{3} \mathbf{S}_{1}$ & 72.10 & 68.18 & 70.14 & 64.30 & - & - & - & - & - & - & - & - & - \\
\hline $\mathrm{T}_{4}-\mathbf{P}_{4} \mathrm{~S}_{1}$ & 73.01 & 70.27 & 71.64 & 67.07 & 63.11 & - & - & - & - & - & - & - & - \\
\hline $\mathrm{T}_{5}-\mathrm{P}_{5} \mathrm{~S}_{1}$ & 71.19 & 66.08 & 68.63 & - & - & - & - & - & - & - & - & - & - \\
\hline $\mathrm{T}_{6}-\mathrm{P}_{1} \mathrm{~S}_{2}$ & 76.83 & 75.10 & 75.96 & 72.26 & 69.01 & 63.53 & 57.42 & 53.50 & 47.63 & 41.23 & - & - & - \\
\hline $\mathrm{T}_{\mathbf{7}}-\mathrm{P}_{2} \mathrm{~S}_{2}$ & 78.12 & 76.42 & 77.27 & 73.80 & 71.86 & 64.38 & 58.71 & 55.46 & 48.52 & 44.42 & 40.74 & 38.73 & - \\
\hline $\mathrm{T}_{8}-\mathbf{P}_{3} \mathrm{~S}_{2}$ & 77.46 & 75.39 & 76.42 & 72.91 & 69.78 & 64.05 & 58.17 & 54.26 & 48.10 & 43.72 & 40.14 & 38.18 & - \\
\hline $\mathrm{T}_{\mathbf{9}}-\mathbf{P}_{4} \mathrm{~S}_{2}$ & 79.85 & 78.11 & 78.98 & 75.10 & 71.22 & 66.56 & 60.24 & 56.83 & 50.82 & 46.83 & 42.75 & 40.89 & 40.12 \\
\hline $\mathrm{T}_{10}-\mathrm{P}_{5} \mathrm{~S}_{2}$ & 76.09 & 74.95 & 75.52 & 71.36 & 68.88 & 62.38 & 56.61 & 52.90 & 46.88 & 40.05 & - & - & - \\
\hline $\mathbf{T}_{11}-\mathbf{P}_{1} \mathbf{S}_{3}$ & 69.46 & - & 34.73 & - & - & - & - & - & - & - & - & - & - \\
\hline $\mathrm{T}_{12}-\mathrm{P}_{2} \mathrm{~S}_{3}$ & 70.09 & 65.11 & 67.60 & - & - & - & - & - & - & - & - & - & - \\
\hline $\mathrm{T}_{13}-\mathrm{P}_{3} \mathrm{~S}_{3}$ & 69.98 & 64.29 & 67.13 & - & - & - & - & - & - & - & - & - & - \\
\hline $\mathrm{T}_{14}-\mathrm{P}_{4} \mathrm{~S}_{3}$ & 70.86 & 65.52 & 68.19 & - & - & - & - & - & - & - & - & - & - \\
\hline $\mathrm{T}_{15}-\mathrm{P}_{5} \mathrm{~S}_{3}$ & 68.62 & - & 34.31 & - & - & - & - & - & - & - & - & - & - \\
\hline $\mathrm{T}_{16}-\mathrm{P}_{1} \mathrm{~S}_{4}$ & 74.02 & 72.85 & 73.43 & 68.73 & 64.55 & 58.78 & - & - & - & - & - & - & - \\
\hline $\mathbf{T}_{17}-\mathbf{P}_{2} \mathrm{~S}_{4}$ & 74.98 & 73.45 & 74.21 & 69.85 & 66.39 & 60.49 & 53.20 & 50.80 & 45.81 & - & - & - & - \\
\hline $\mathrm{T}_{18}-\mathrm{P}_{3} \mathrm{~S}_{4}$ & 74.42 & 73.00 & 73.71 & 69.20 & 66.07 & 60.04 & 52.48 & 50.12 & - & - & - & - & - \\
\hline $\mathrm{T}_{19}-\mathrm{P}_{4} \mathrm{~S}_{4}$ & 75.86 & 74.16 & 75.01 & 70.52 & 68.13 & 62.09 & 55.76 & 52.08 & 46.21 & - & - & - & - \\
\hline $\mathrm{T}_{20}-\mathrm{P}_{5} \mathrm{~S}_{4}$ & 73.58 & 72.23 & 72.90 & 67.98 & 63.80 & 57.12 & - & - & - & - & - & - & - \\
\hline Mean & 73.55 & 64.02 & & & & & & & & & & & \\
\hline
\end{tabular}

\begin{tabular}{|c|}
\hline Factors \\
\hline For treatments (T) \\
\hline For days (D) \\
\hline Factor(T×D) \\
\hline *Significant at $(\mathbf{P} \leq \mathbf{0 . 0 5})$ \\
\hline
\end{tabular}

\begin{tabular}{|c|c|}
\hline C.D at 5\% & SE(d) \\
\hline 1.910 & 0.958 \\
\hline 0.604 & 0.303 \\
\hline 2.702 & 1.355 \\
\hline
\end{tabular}

$\mathrm{S}_{1}$ : Ambient temperature $\left(22 \pm 2^{\circ} \mathrm{c}\right)$ (Wet storage) $\mathrm{P}_{2}$ : Polyethylene (100 gauge) $\mathrm{P}_{3}$ : Cellophane paper

$\mathrm{P}_{4}$ : Polypropylene $\mathrm{P}_{5}$ : Control (open)
$\mathrm{S}_{2}$ : Cold storage of $5^{\circ} \mathrm{C}$ (Wet storage)

$\mathrm{S}_{3}$ : Ambient temperature $\left(22 \pm 2^{\circ} \mathrm{c}\right)$ (Dry storage)

$\mathrm{S}_{4}$ : Cold storage of $5^{\circ} \mathrm{C}$ (Dry storage) 
Table.2 Effect of different packaging and storage conditions on chlorophyll content of calyx (SPAD units) during vase life of cut carnation (Dianthus caryophyllus L.) cv. Kiro

\begin{tabular}{|c|c|c|c|c|c|c|c|c|c|c|c|c|c|}
\hline \multirow[t]{2}{*}{ Treatments } & \multicolumn{13}{|c|}{ Days } \\
\hline & 2 & 4 & Mean & 6 & 8 & 10 & 12 & 14 & 16 & 18 & 20 & 22 & 24 \\
\hline $\mathrm{T}_{1}-\mathrm{P}_{1} \mathrm{~S}_{1}$ & 26.94 & 24.38 & 25.66 & 20.85 & - & - & - & - & - & - & - & - & - \\
\hline $\mathbf{T}_{2}-\mathbf{P}_{2} \mathbf{S}_{1}$ & 28.05 & 25.83 & 26.94 & 23.91 & - & - & - & - & - & - & - & - & - \\
\hline $\mathrm{T}_{3}-\mathrm{P}_{3} \mathrm{~S}_{1}$ & 27.63 & 25.03 & 26.33 & 22.83 & - & - & - & - & - & - & - & - & - \\
\hline $\mathbf{T}_{4}-\mathbf{P}_{4} \mathbf{S}_{1}$ & 28.83 & 26.42 & 27.62 & 24.60 & 23.22 & - & - & - & - & - & - & - & - \\
\hline $\mathbf{T}_{5}-\mathbf{P}_{5} \mathrm{~S}_{1}$ & 26.25 & 24.01 & 25.13 & - & - & - & - & - & - & - & - & - & - \\
\hline $\mathrm{T}_{6}-\mathrm{P}_{1} \mathrm{~S}_{2}$ & 34.82 & 33.56 & 34.19 & 31.42 & 29.70 & 26.96 & 25.45 & 23.78 & 21.98 & 18.85 & - & - & - \\
\hline $\mathbf{T}_{7}-\mathbf{P}_{2} \mathbf{S}_{2}$ & 36.77 & 35.17 & 35.97 & 33.40 & 31.20 & 29.35 & 26.91 & 25.24 & 23.82 & 21.69 & 20.46 & 17.41 & - \\
\hline $\mathbf{T}_{8}-\mathbf{P}_{3} \mathbf{S}_{2}$ & 35.17 & 34.42 & 34.79 & 32.51 & 30.19 & 28.68 & 26.10 & 24.49 & 22.74 & 20.48 & 18.21 & 16.48 & - \\
\hline $\mathrm{T}_{9}-\mathbf{P}_{4} \mathrm{~S}_{2}$ & 38.89 & 37.12 & 38.00 & 35.65 & 33.47 & 31.85 & 29.77 & 27.15 & 25.60 & 23.42 & 21.37 & 19.30 & 18.25 \\
\hline $\mathrm{T}_{10}-\mathrm{P}_{5} \mathrm{~S}_{2}$ & 34.06 & 32.91 & 33.48 & 30.86 & 28.62 & 26.17 & 24.82 & 23.07 & 20.89 & 18.13 & - & - & - \\
\hline $\mathrm{T}_{11}-\mathrm{P}_{1} \mathrm{~S}_{3}$ & 24.20 & - & 12.10 & - & - & - & - & - & - & - & - & - & - \\
\hline $\mathrm{T}_{12}-\mathrm{P}_{2} \mathrm{~S}_{3}$ & 25.58 & 22.52 & 24.05 & - & - & - & - & - & - & - & - & - & - \\
\hline $\mathrm{T}_{13}-\mathrm{P}_{3} \mathrm{~S}_{3}$ & 25.11 & 20.61 & 22.86 & - & - & - & - & - & - & - & - & - & - \\
\hline $\mathrm{T}_{14}-\mathrm{P}_{4} \mathrm{~S}_{3}$ & 25.99 & 23.43 & 24.71 & - & - & - & - & - & - & - & - & - & - \\
\hline $\mathrm{T}_{15}-\mathrm{P}_{5} \mathrm{~S}_{3}$ & 23.53 & - & 11.76 & - & - & - & - & - & - & - & - & - & - \\
\hline $\mathrm{T}_{16}-\mathrm{P}_{1} \mathrm{~S}_{4}$ & 30.29 & 29.18 & 29.73 & 24.67 & 24.67 & 24.03 & - & - & - & - & - & - & - \\
\hline $\mathrm{T}_{17}-\mathrm{P}_{2} \mathrm{~S}_{4}$ & 32.90 & 30.69 & 31.80 & 27.42 & 26.30 & 25.11 & 22.90 & 20.26 & 18.090 & - & - & - & - \\
\hline $\mathrm{T}_{18}-\mathrm{P}_{3} \mathrm{~S}_{4}$ & 31.88 & 29.81 & 30.84 & 26.77 & 25.42 & 24.50 & 22.09 & 20.04 & - & - & - & - & - \\
\hline $\mathrm{T}_{19}-\mathrm{P}_{4} \mathrm{~S}_{4}$ & 33.75 & 31.75 & 32.75 & 29.70 & 28.12 & 25.87 & 23.70 & 22.35 & 18.947 & - & - & - & - \\
\hline $\mathrm{T}_{20}-\mathrm{P}_{5} \mathrm{~S}_{4}$ & 28.90 & 28.67 & 28.78 & 25.89 & 24.16 & 23.28 & - & - & - & - & - & - & - \\
\hline Mean & 29.97 & 25.77 & & & & & & & & & & & \\
\hline
\end{tabular}

\begin{tabular}{|c|}
\hline Factors \\
\hline For treatments (T) \\
\hline For days (D) \\
\hline Factor $(\mathrm{T} \times \mathrm{D})$ \\
\hline *Significant at $(\mathbf{P} \leq \mathbf{0 . 0 5})$ \\
\hline
\end{tabular}

C.

\begin{tabular}{|c|c|c|c|}
\hline C.D at 5\% & SE d & P \\
\hline 0.865 & 0.434 & P \\
\hline 0.273 & 0.137 & $\mathrm{P}$ \\
\hline 1.223 & 0.613 & $\mathrm{P}$ \\
\hline & & $\mathrm{P}$ \\
\hline
\end{tabular}

\section{$\mathbf{P}_{1}$ : PVC cling film}

$\mathrm{P}_{2}$ : Polyethylene (100 gauge)

$\mathrm{P}_{3}$ : Cellophane paper

$\mathrm{P}_{4}$ : Polypropylene

$\mathrm{P}_{5}$ : Control (open)
$\mathrm{S}_{1}$ : Ambient temperature $\left(22 \pm 2^{\circ} \mathrm{c}\right)$ (Wet storage)

$\mathrm{S}_{2}$ : Cold storage of $5^{\circ} \mathrm{C}$ (Wet storage)

$\mathrm{S}_{3}$ : Ambient temperature $\left(22 \pm 2^{\circ} \mathrm{c}\right)$ (Dry storage)

$\mathrm{S}_{4}$ : Cold storage of $5^{\circ} \mathrm{C}$ (Dry storage) 
Table.3 Effect of different packaging and storage conditions on ethylene estimation $(\mathrm{ppm} / \mathrm{h})$ during vase life of cut carnation (Dianthus caryophyllus L.) cv. Kiro

\begin{tabular}{|c|c|c|c|c|c|c|c|c|c|c|c|c|c|}
\hline \multirow[t]{2}{*}{ Treatments } & \multicolumn{13}{|c|}{ Days } \\
\hline & 2 & 4 & Mean & 6 & 8 & 10 & 12 & 14 & 16 & 18 & 20 & 22 & 24 \\
\hline $\mathrm{T}_{1}-\mathrm{P}_{1} \mathrm{~S}_{1}$ & 4.90 & 6.96 & 5.93 & 7.22 & - & - & - & - & - & - & - & - & - \\
\hline $\mathrm{T}_{2}-\mathbf{P}_{2} \mathrm{~S}_{1}$ & 4.35 & 6.00 & 5.18 & 6.65 & - & - & - & - & - & - & - & - & - \\
\hline $\mathrm{T}_{3}-\mathrm{P}_{3} \mathrm{~S}_{1}$ & 4.90 & 6.21 & 5.55 & 6.87 & - & - & - & - & - & - & - & - & - \\
\hline $\mathrm{T}_{4}-\mathrm{P}_{4} \mathrm{~S}_{1}$ & 4.03 & 5.91 & 4.97 & 6.41 & 7.50 & - & - & - & - & - & - & - & - \\
\hline $\mathrm{T}_{5}-\mathrm{P}_{5} \mathrm{~S}_{1}$ & 5.01 & 7.15 & 6.08 & - & - & - & - & - & - & - & - & - & - \\
\hline $\mathrm{T}_{6}-\mathrm{P}_{1} \mathrm{~S}_{2}$ & 1.85 & 2.87 & 2.36 & 3.15 & 3.91 & 4.94 & 5.36 & 5.59 & 6.93 & 7.51 & - & - & - \\
\hline $\mathrm{T}_{7}-\mathrm{P}_{2} \mathrm{~S}_{2}$ & 0.84 & 1.19 & 1.02 & 1.97 & 2.67 & 3.72 & 4.58 & 4.91 & 5.80 & 6.45 & 7.35 & 9.16 & - \\
\hline $\mathrm{T}_{8}-\mathbf{P}_{3} \mathrm{~S}_{2}$ & 1.29 & 2.09 & 1.69 & 2.88 & 3.58 & 4.10 & 5.00 & 5.17 & 6.09 & 6.96 & 7.97 & 10.52 & - \\
\hline $\mathrm{T}_{9}-\mathrm{P}_{4} \mathrm{~S}_{2}$ & 0.21 & 0.33 & 0.27 & 0.92 & 1.35 & 2.47 & 3.35 & 3.97 & 4.47 & 5.14 & 6.20 & 7.80 & 10.12 \\
\hline $\mathrm{T}_{10}-\mathrm{P}_{5} \mathrm{~S}_{2}$ & 2.09 & 3.11 & 2.60 & 3.84 & 4.26 & 5.08 & 5.92 & 6.12 & 7.05 & 7.70 & - & - & - \\
\hline $\mathrm{T}_{11}-\mathrm{P}_{1} \mathrm{~S}_{3}$ & 7.42 & - & 3.71 & - & - & - & - & - & - & - & - & - & - \\
\hline $\mathrm{T}_{12}-\mathrm{P}_{2} \mathrm{~S}_{3}$ & 5.89 & 8.12 & 7.00 & - & - & - & - & - & - & - & - & - & - \\
\hline $\mathbf{T}_{13}-\mathbf{P}_{3} \mathbf{S}_{3}$ & 6.55 & 8.43 & 7.49 & - & - & - & - & - & - & - & - & - & - \\
\hline $\mathrm{T}_{14}-\mathrm{P}_{4} \mathrm{~S}_{3}$ & 5.22 & 7.89 & 6.55 & - & - & - & - & - & - & - & - & - & - \\
\hline $\mathrm{T}_{15}-\mathrm{P}_{5} \mathrm{~S}_{3}$ & 7.90 & - & 3.95 & - & - & - & - & - & - & - & - & - & - \\
\hline $\mathrm{T}_{16}-\mathrm{P}_{1} \mathrm{~S}_{4}$ & 3.47 & 5.10 & 4.28 & 5.19 & 5.47 & 6.05 & - & - & - & - & - & - & - \\
\hline $\mathrm{T}_{17}-\mathrm{P}_{2} \mathrm{~S}_{4}$ & 2.97 & 4.05 & 3.51 & 4.21 & 4.98 & 5.56 & 6.39 & 6.56 & 7.66 & - & - & - & - \\
\hline $\mathrm{T}_{18}-\mathrm{P}_{3} \mathrm{~S}_{4}$ & 3.10 & 4.76 & 3.93 & 5.03 & 5.15 & 5.88 & 6.94 & 7.17 & - & - & - & - & - \\
\hline $\mathrm{T}_{19}-\mathrm{P}_{4} \mathrm{~S}_{4}$ & 2.53 & 3.85 & 3.19 & 4.07 & 4.45 & 5.20 & 6.12 & 6.30 & 7.25 & - & - & - & - \\
\hline $\mathrm{T}_{20}-\mathrm{P}_{5} \mathrm{~S}_{4}$ & 3.91 & 5.35 & 4.63 & 5.86 & 6.32 & 6.62 & - & - & - & - & - & - & - \\
\hline Mean & 3.92 & 4.47 & & & & & & & & & & & \\
\hline
\end{tabular}

\begin{tabular}{|c|}
\hline Factors \\
\hline For treatments (T) \\
\hline For days (D) \\
\hline Factor $(\mathrm{T} \times \mathrm{D})$ \\
\hline *Significant at $(\mathbf{P} \leq \mathbf{0 . 0 5})$ \\
\hline
\end{tabular}

\begin{tabular}{|c|}
\hline C.D at $5 \%$ \\
\hline 0.106 \\
\hline 0.034 \\
\hline 0.150 \\
\hline
\end{tabular}

\begin{tabular}{|l|}
\hline SE d \\
\hline 0.053 \\
0.017 \\
0.075
\end{tabular}

\begin{tabular}{|l}
$\mathrm{P}_{1}: \mathbf{P}$ \\
$\mathrm{P}_{2}: \mathrm{Pr}$ \\
$\mathrm{P}_{3}: \mathrm{C}$ \\
$\mathrm{P}_{5}: \mathrm{C}$
\end{tabular}

$\mathrm{S}_{1}$ : Ambient temperature $\left(22 \pm 2^{\circ} \mathrm{c}\right)$ (Wet storage)

$\mathrm{S}_{2}$ : Cold storage of $5^{\circ} \mathrm{C}$ (Wet storage)

$\mathrm{S}_{3}$ : Ambient temperature $\left(22 \pm 2^{\circ} \mathrm{c}\right)$ (Dry storage)

$\mathrm{S}_{4}$ : Cold storage of $5^{\circ} \mathrm{C}$ (Dry storage) 
These results were in accordance with Genkov et al., (1997), Misson et al., (2000), Sudha et al., (2000) and Madhuri et al., (2016) in carnation.

The ethylene estimation was significant among all the treatment combinations. The lowest ethylene estimation $(0.27 \mathrm{ppm} / \mathrm{h})$ was recorded with flowers packed in polypropylene $5^{\circ} \mathrm{C}$ cold storage under wet condition $\left(\mathrm{T}_{9}\right)$. Cellophane paper packed flowers at ambient temperature $\left(22 \pm 2^{\circ} \mathrm{C}\right)$ under dry condition $\left(\mathrm{T}_{13}\right)$ recorded significantly the highest ethylene estimation $(7.49 \mathrm{ppm} / \mathrm{h})$ whereas, the remaining all other treatments recorded intermediate values. There were significant differences in ethylene estimation during different days of vase life period. The ethylene estimation significantly increased from $2^{\text {nd }}$ day $(3.92 \mathrm{ppm} / \mathrm{h})$ to $4^{\text {th }}$ day (4.47 $\mathrm{ppm} / \mathrm{h}$ ) at each successive interval of observation. The interaction effect between treatments and days on ethylene estimation was found significant. The treatment $\mathrm{T}_{9}$ flowers packed in polypropylene at $5^{\circ} \mathrm{C}$ cold storage under wet condition recorded significantly the lowest ethylene estimation $(0.21 \mathrm{ppm} / \mathrm{h})$ on $2^{\text {nd }}$ day followed by flowers packed in polyethylene (100 gauge) at $5^{\circ} \mathrm{C}$ cold storage under wet condition $\left(\mathrm{T}_{7}\right)(0.84$ $\mathrm{ppm} / \mathrm{h})$. On $4^{\text {th }}$ day polypropylene packed flowers at $5^{\circ} \mathrm{C}$ cold storage under wet condition $\left(\mathrm{T}_{9}\right)$ recorded the lowest ethylene estimation $(0.33 \mathrm{ppm} / \mathrm{h})$ followed by $\left(\mathrm{T}_{7}\right)$ i.e polyethylene (100 gauge) packed flowers at $5^{\circ} \mathrm{C}$ cold storage under wet condition (1.19 $\mathrm{ppm} / \mathrm{h}$ ). Polypropylene packed flowers at $5^{\circ} \mathrm{C}$ cold storage under wet condition $\left(\mathrm{T}_{9}\right)$ recorded the lowest ethylene estimation on all the days of vase life study. The data indicated that lowest ethylene estimation in polypropylene packed flowers kept in cold storage under wet condition might be due to polypropylene created congenial microclimate around the flower tissues so it ensure membrane integrity and would extend the vase life. The positive effect of cold storage results in low ethylene release due to low metabolic activities like respiration, transpiration and maintains high humidity under wet condition. Similar results were observed by Pun et al., (2005) in spray carnation, Ichimura et al., (2009) in cut eustoma, delphinium and snapdragon and Muchui (2012) in banana.

\section{References}

Bhattacharjee, S.K. 1999. Postharvest life and biochemical constituents of Sonia Meilland cut roses as affected by chloride salts. Indian Agriculturist. 43(1/2): 1-10.

Genkov, T., Tsoneva, P. and Ivanova, I. 1997. Effect of cytokinins on photosynthetic pigments and chlorophyllase activity in in vitro cultures of axillary buds of (Dianthus caryophyllus L.). Journal of Plant Growth Regulation. 16(3): 169172.

Halevy, A.H. and Mayak, S. 1981. Senescence and postharvest physiology of cut flowers. Part I. In: Horticultural Reviews. Vol. 2, AVI Publishing Westport, conn. 59-143.

Ichimura, K., Yamada, T. and Shimizu, Y. H. 2009. Recent breakthroughs in postharvest physiology research and cut flower handling in Japan. Horticulture Environmental Biotechnology. 50(6): 539-545.

Lavanya, V., Nidoni, U. R., Kurubar, A.R., Sharanagouda, H. and Ramachandra, C.T. 2016. Effect of pre-treatment and different packaging materials on shelflife of Jasmine flowers (Jasmine sambac). Environment \& Ecology. 34(1A): 341-345.

Madhuri, M., Bindu, P. R., Hari, P. R. N. and vijay, I. 2016. Effect of biocides on the fresh weight, chrolophyll content and microbial load in cut carnation flowers 
cv. Charmant. Plant Archives. 16(2), pp. 777-780.

Misson, B., Sang, C., Kia, K., Byun, M. S., Sang, C. K. and Kim, K.W. 2000. Flowering response of cut carnation harvested at various bud stages and stored at low temperature. Journal of the Korean Society for Horticultural Science, 41(5): 531-534.

Muchui, M.N. 2012. Influence of fertilizers, harvest maturity, polyethylene bunch covers and postharvest treatment with 1-Methylcyclopropene on physical, physiological and biochemical quality of tissue-cultured bananas (Musa spp.) A thesis submitted in fulfillment for the degree of Doctor of Philosophy in Food Science and Postharvest Technology in the Jomo Kenyatta University of Agriculture and Technology.
Pun, U. K., Shimizu, H., Tanase, K. and Ichimura, K. 2005. Effect of sucrose on ethylene biosynthesis in cut spray carnation flowers. Acta Horticulturae. 669: 171-174.

Singh, A. K. 2006. Flower crops cultivation and management. New India publishing agency. Pitam Pura, New Delhi.

Sivaswamy. N., Sujatha, A. N., Attri, B. L. and Sharma, T. V. R. S. 1999. Postharvest technology of cut flowers. Agro India. 4: 12-13.

Sudha, U. 2010. Studies on the effect of temperature, sucrose, organic acids, biocides and packing on extension of vase life of carnation (Dianthus caryophyllus L.) cv. Domingo. M. Sc. Thesis submitted to Dr. Y.S.R. Horticultural University, Rajendranagar.

\section{How to cite this article:}

Pranuthi, P., T. Suseela, D.V. Swami, D.R. Salomi Suneetha and Sudha Vani, V. 2018. Effect of Different Packing and Storage Conditions on Biochemical Parameters in Extending the Vase Life of Cut Carnation cv. Kiro. Int.J.Curr.Microbiol.App.Sci. 7(08): 1348-1355. doi: https://doi.org/10.20546/ijcmas.2018.708.153 